\title{
Making malaria research bite
}

An audit of international activity in malaria research highlights the need for a more concerted research effort.

In the time it takes you to read this article 23 people will have died of malaria. The disease threatens 40 percent of the world's population - 2400 million peo-

\section{Marlie MacLean, Joe Anderson} \& CATHERINE DAVIES

ple - in more than 90 countries. It causes an estimated 300-500 million clinical cases and 1.5-2.7 million deaths per year'. If these figures alone aren't worrying enough, incidences of the disease are on the increase. Efforts to develop a malaria vaccine have so far been unsuccessful, and the effectiveness of most antimalarial drugs has greatly decreased. However, a recent study has shown that total identifiable global expenditure on malaria research is markedly less than on some other major diseases. Furthermore, while research contributes to improvements in health, much more can be done to put the results of scientific investigation to work. An international survey of malaria experts highlights the barriers to exploiting research results, identifies possible measures to tackle those obstacles, and assesses the prospects of various subfields of research for advancing knowledge over the next five years.

\section{Current disease situation}

The Plasmodium parasite is becoming increasingly resistant to antimalarial drugs, including multidrug cocktails, in several countries $^{2}$. Control has become more difficult because of insecticide resistance of the Anopheles mosquito vectors and because of activities such as mining, changing agricultural practices, armed conflict and refugee movements' ${ }^{1}$. Furthermore, recent trials of an experimental vaccine have not been encouraging, and severe side effects from the use of the relatively new drug mefloquine (trade name Lariam) have been identified ${ }^{+}$.

Malaria is principally a disease of less developed tropical countries in Africa, Asia and Central and South America. The governments of many of these countries spend their limited resources fighting disease at the front line, leaving little or no money to invest in medical research. Pharmaceutical firms are aware both of the expense of developing new antimalarial drugs and vaccines and of their limited financial reward: who, after all, will buy pharmaceutical products for the world's poor?

\section{Funding research}

There is a pressing need for research in the field of malaria and for the effective translation of research results into practical application. A study published recently by the Unit for Policy Research in Science and Medicine (PRISM) at the Wellcome Trust has estimated that total identifiable global expenditure on malaria research in 1993, from both public and nonprofit sectors, was only about $\$ 84$ million $^{5}$. Although data on investment from industry were not available, it is evident that there are a limited number of companies active in this area.

International malaria research expenditure equates to approximately $\$ 42$ for every death worldwide (based on a figure of 2 million deaths per year). In other disease areas, estimates for global annual expenditure on research and development were \$919-985 million for HIV/AIDS and \$127-158 million for asthma (over the period 1990-1992 and including the pharma- ceutical industry $)^{6}$. Based on global mortality figures from the literature, expenditure per fatal case can be calculated as $\$ 3274$ for HIV/AIDS and $\$ 789$ for asthmas. These figures are one to two orders of magnitude greater than the equivalent malaria figure, and they indicate that global research funding disproportionately prioritizes high-profile diseases in the West.

International organizations, philanthropic foundations and governments of some developed countries are the major funders of malaria research. The PRISM report revealed that more than half of the 1993 funding came from the USA, particularly the US Agency for International Development (USAID) and the National Institute of Allergy and Infectious Diseases (NIAID). Much of the remainder came from Europe - notably from the United Kingdom where the Wellcome Trust and the Medical Research Council (MRC) are very active - and from the Programme in Research and Training in Tropical Diseases (TDR). The TDR Programme is cosponsored by the United Nations Development Programme, the World Bank and the World Health Organization (WHO) as well as several governments, especially in Scandinavia.

The trend in worldwide funding over the last decade has been downward, largely reflecting decreasing investment from the USA, especially USAID. Meanwhile, malaria research funding from the TDR Programme has remained relatively constant as the UK has increased its contribution. Clearly, the UK has an increasing presence in the funding of malaria research. But what have been the results and broader outcomes from these investments?

\section{Publication of research results}

An analysis by PRISM revealed that the number of international papers in malaria published per year is only about 1000 , a figure that mirrors the low funding. Furthermore, publication output from different countries and funding bodies largely reflected the balance of funding. The USA is currently the largest single contributor to the malaria research literature, but its share of world publications has declined over the past ten years (from 42 percent in 1984 to 34 percent in 1994). Meanwhile, the UK contribution has moved from 14 percent to 18 percent of the total world publications concerning malaria. These figures indicate the strong UK presence in the field of malaria relative to other biomedical fields where the UK share of global publications ranged from $6.9 \%$ to $16.1 \%$ (ref. 8). Although this analysis excludes literature published in national and nonEnglish language journals, it clearly identifies the international players. All well and good, but what are the broader impacts of the research?

\section{Health-care impacts}

In a survey of more than 100 malaria experts, PRISM canvassed the opinions of both researchers and users on the role of malaria research in health care. Included in the survey were scientists, health services practitioners/advisers, administrators/policy makers and industrialists/manufacturers in both malaria- 
endemic and nonendemic countries. The survey showed that more than two-thirds of respondents had personal experience of projects where research results were developed to improve disease prevention, treatment or control - a very high percentage, indicating the direct impact of malaria research on health care. Furthermore, the survey also indicated that the benefits of research were largely expected within the relatively short time span of $1-5$ years.

Respondents' experiences of the practical use of results mainly focused on involvement in some stage of the development and testing of antimalarial drugs, in optimization of treatment or prophylactic drug dosage regimens, or in improving methods for the clinical management of malaria. Experience in developmental projects was also frequently cited in research related to intervention trials and health services research. Other respondents indicated research experience leading to vaccine development and vaccine trials.

However, the most worrying aspect of the survey was the

Prospects in each research subfield, expressed as a percentage of survey responses, for substantial advances in understanding over the next five years ( $n=$ total number of responses).

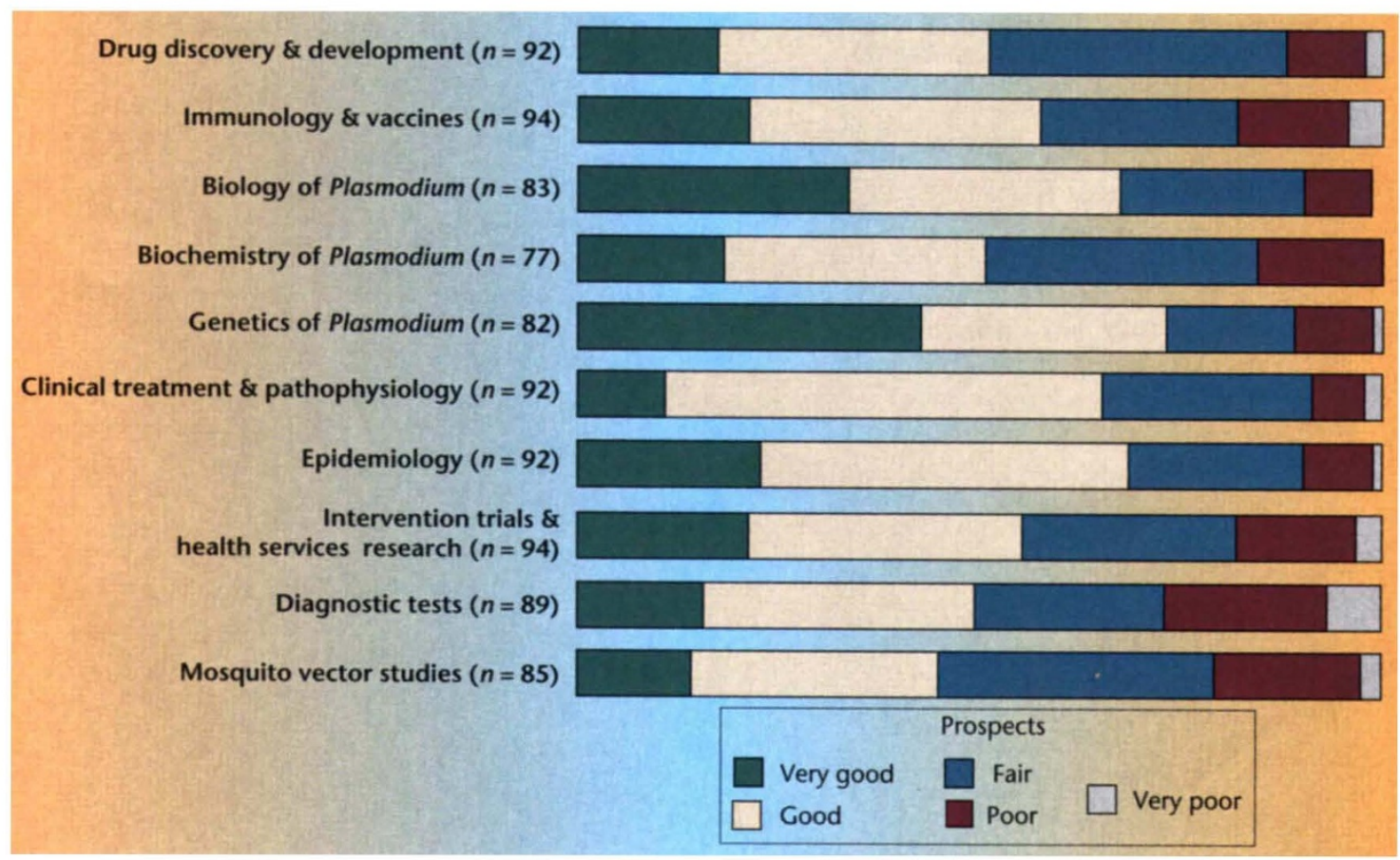

for making substantial advances in understanding. Three other subfields were also perceived as having very good prospects for advancing knowledge in the medium term: epidemiology and mathematical modeling; intervention trials and health services research; and immunology and vaccine development. Some respondents also anticipated that the probable sequencing of the malaria genome within the next five years may lead to entirely new opportunities.

A review of the recent scientific literature identifies a number of promising developments for the practical control or prevention of malaria. Encouraging results have been published in operational research where the use of bed nets treated with the insecticide permethrin reduced childhood mortality by $33 \%$ and the risk of developing severe, life-threatening malaria in children by $44 \%$ (ref. 10). In the area of drug development, the use of wormwood (Artemisia)-derived artemether afforded a similar survival rate of patients with cerebral malaria as quinine, but caused less irritation and cleared the parasite from the body more quickly ${ }^{11,12}$. Another development is the genetically engi- 
neered mosquito designed to transmit selected proteins and to encourage the production of antibodies against Plasmodium ${ }^{13}$. Finally, results published in this issue of Nature Medicine (pp. 80-83) indicate that injection of monkeys with recombinant human interleukin-12 can stimulate the immune system and protect against malaria ${ }^{14}$. Further studies of this factor for immunoprophylaxis of human malaria appear warranted.

\section{The need for more concerted research effort}

Scientific research underpins improvements in health care. Some international organizations, charities and governments are actively engaged in malaria research, but it is clear that more public investment is required. In addition, incentives to encourage investment by the pharmaceutical industry are needed. The renewal of the "Orphan Drug Tax Credits" in the USA (Public Law 104-188, 20 August 1996) allows American companies to deduct half of the costs of clinical testing of drugs that target diseases that are rare in the American population ${ }^{15}$. It is hoped that this law will encourage industrial investment in malaria research and will raise the issue of whether other countries should introduce similar measures to encourage industrial research and development.

In the meantime, the incidence of malaria has moved upward. The number of cases in Eastern Europe is increasing rapidly as a result of the economic collapse in countries of the former Soviet Union ${ }^{16}$, and both international migration and travel have caused mortalities in the United Kingdom ${ }^{17}$. In addition, global climate change has the potential to alter the distribution of vector-borne diseases, potentially causing 50-80 million additional cases of malaria per year ${ }^{18}$. These developments make it even more urgent that greater attention be paid to malaria research and to translating the results of such investigation into practice.

\section{Acknowledgments}

The authors would like to thank Robert Howells and members of both the Tropical Medicine Interest Group and the Malaria Audit Steering Committee of the Wellcome Trust for their guidance and contribution. The work was funded by the Wellcome Trust.
1. World Health Organization. The World Health Report 1996. (World Health Organization, Geneva, 1996)

2. Bruce-Chwatt, L.J. Essential Malariology. (Heinemann Medical Books Ltd, London, 1987).

3. Nosten, F. et al. Randomised double-blind placebo-controlled trial of Spf66 malaria vaccine in children in northwestern Thailand. Lancet 348, 701-707 (1996).

4. Barrett, P.J., Emmins, P.D., Clarke, P.D. \& Bradley, D.J. Comparison of adverse events associated with use of mefloquine and combination of chloroquine and proguanil as antimalarial prophylaxis: Postal and telephone survey of travellers. Br. Med. I. 313, 525-528 (1996).

5. Anderson, J., MacLean, M. \& Davies, C. Malaria research: An audit of international activity. (PRISM report no. 7, Wellcome Trust, London, 1996).

6. Michaud, C. \& Murray, C.J.L. Resources for health research and development in 1992: A global overview. in Investing in Heaith Research and Development. (Ad Hoc Committee on Heaith Research Relating to Future intervention Options, World Health Organization, TDR/Gen/96.1, Geneva, 1996)

7. Murray, C.J.L. \& Lopez, A.D. (eds) Global Comparative Assessments in the Health Sector: Disease Burden, Expenditures and intervention Packages. (World Health Organization, Geneva, 1996).

8. Lewison, C. \& Seemungal, D. Benchmarking information: Research investment and research output. Companion paper A to the Office of Science and Technology Health and Life Sciences Report. (Office of Science and Technology, Office of Public Service and Science, Cabinet Office, London, 1995).

9. Garner, P., Zaat, J. \& Robb, R. Tropical medicine on trial: Getting hold of all the evidence. Parasitol. Today 12, 211-212 (1996).

10. Neville, C.G. et al. Insecticide-treated bednets reduce mortality and severe morbidity from malaria among children on the Kenyan coast. I. Trop. Med. Intl. Health 1, 139-146 (1996).

11. Boele van Hensbroek, M. et al. A trial of artemether or quinine in children with cerebral malaria. N. Engl. /. Med. 335, 69-75 (1996).

12. Tran, T.H. et al. A controlled trial of artemether or quinine in Vietnamese adults with severe falciparium malaria. N. Engl. J. Med. 335, 76-83 (1996).

13. Sinden, R.E. \& Crampton, J.M. International patent application, WO 96/15237, delivery system.

14. Hoffman, S.L. et al. Sterile protection of monkeys against malaria after administration of interleukin-12. Nature Med. 3, 80-83 (1996).

15. Wadman, M. Budget politics jeopardizes US drug credit. Nature 379, 287 (1996).

16. Tuberculosis reappears in Europe. Nature 380, 99 (1996).

17. Bradley, D., Warhurst, D., Blaze, M. \& Smith, V. Malaria imported into the United Kingdom in 1992 and 1993. Communicable Disease Report, CDR Review 4, 169-172 (1994).

18. McMichael, A.J., Haines, A., Sloof, R., \& Kovats, S. Climate Change and Human Health. Geneva. (World Health Organization, Geneva, 1996).

The Unit for Policy Research in Science and Medicine (PRISM),

The Wellcome Trust, 210 Euston Road

London NW1 2BE, UK

M.M. present address: Biotechnology and Biological Sciences Research Council (BBSRC),

Polaris House, North Star Avenue,

Swindon, SN2 1UH, UK 